\title{
DZNep, an inhibitor of the histone methyltransferase EZH2, suppresses hepatic fibrosis through regulating miR-199a-5p/SOCS7 pathway
}

\author{
Rongrong Ding ${ }^{1,2}$, Jianming Zheng ${ }^{1}$, Ning Li ${ }^{1}$, Qi Cheng ${ }^{1}$, Mengqi Zhu ${ }^{1}$, Yanbing Wang ${ }^{2}$, Xinlan Zhou ${ }^{2}$, Zhanqing \\ Zhang ${ }^{\text {Corresp., } 2}$, Guangfeng Shi ${ }^{\text {Corresp. } 1}$ \\ 1 Infectious Diseases, Huashan Hospital, Fudan University, Shanghai, China \\ 2 Hepatobiliary Medicine, Shanghai Public Health Clinical Center, Fudan University, Shanghai, China \\ Corresponding Authors: Zhanqing Zhang, Guangfeng Shi \\ Email address: doctorzzqsphc@163.com,gfshi2005@163.com
}

Background. Hepatic fibrosis is a common response to chronic liver injury. Recently , the role of DZNep (a histone methyltransferase EZH2 inhibitor) in repressing pulmonary and renal fibrosis was verified. However, the potential effect of DZNep on hepatic fibrosis has not been elucidated. Methods. The hepatic fibrosis model was established in rats treated with CCl4 and in hepatic stellate cells (HSCs) treated with TGF- $\beta 1$. The liver tissues were stained with H\&E and Masson's trichrome. The expression of EZH2, SOCS7, collagen I, $\alpha \square S M A$ mRNA and miR-199a-5p was assessed using qPCR, immunohistochemical or western blot analysis. A dual-luciferase reporter assay was carried out to validate the regulatory relationship of miR-199a-5p with SOCS7. Results. The EZH2 level was increased in CCl4-treated rats and in TGF- $\beta 1$-treated HSCs, whereas DZNep treatment significantly inhibited EZH2 expression. DZNep repressed hepatic fibrosis in vivo and in vitro, as evidenced by the decrease of hepatic fibrosis markers ( $\alpha$-SMA and Collagen I). Moreover, miR-199a-5p expression was repressed by DZNep in TGF- $\beta 1$-activated HSCs. Notably, downregulation of miR-199a-5p decreased TGF- $\beta 1$-induced expression of fibrosis markers. SOCS7 was identified as a direct target of miR-199a-5p. The expression of SOCS7 was decreased in TGF- $\beta 1$-activated HSCs, but DZNep treatment restore d SOCS7 expression. More importantly, SOCS7 knockdown decreased the effect of DZNep on collagen I and $\alpha \square S M A$ expression in TGF- $\beta 1$-activated HSCs. Conclusions. DZNep suppresses hepatic fibrosis through regulating miR-199a-5p/SOCS7 axis, suggesting that DZNep may represent a novel treatment for fibrosis. 
1 DZNep, an inhibitor of the histone methyltransferase EZH2, alleviates hepatic

4 Rongrong Ding ${ }^{1,2}$, Jianming Zheng ${ }^{1}$, Ning Li ${ }^{1}$, Qi Cheng ${ }^{1}$, Mengqi Zhu ${ }^{1}$, Yanbing Wang ${ }^{2}$, Xinlan 5 Zhou ${ }^{2}$, Zhanqing Zhang ${ }^{2}$, *, Guangfeng Shi ${ }^{1,}$ *

$7 \quad{ }^{1}$ Infectious Diseases, Huashan Hospital, Fudan University, Shanghai, China.

$8 \quad{ }^{2}$ Hepatobiliary Medicine, Shanghai Public Health Clinical Center, Fudan University, Shanghai, 9 China.

*Correspondence to: Guangfeng Shi, MD, PhD, Huashan Hospital, Fudan University, NO.12

12 Wurumqi Middle Road, Jing'an district, Shanghai 200040, China. Email: gfshi2005@163.com. Zhanqing Zhang, MD, Shanghai Public Health Clinical Center, Fudan University, Caolang Road 2901, Jinshan District, Shanghai 201508, China. Email: doctorzzqsphc@163.com 
Background. Hepatic fibrosis is a common response to chronic liver injury. Recently, the role of DZNep (a histone methyltransferase EZH2 inhibitor) in repressing pulmonary and renal fibrosis was verified. However, the potential effect of DZNep on hepatic fibrosis has not been elucidated.

Methods. The hepatic fibrosis model was established in rats treated with $\mathrm{CCl} 4$ and in hepatic stellate cells (HSCs) treated with TGF- $\beta 1$. The liver tissues were stained with H\&E and Masson's trichrome. The expression of EZH2, SOCS7, collagen I, $\alpha$-SMA mRNA and miR-199a-5p was assessed using qPCR, immunohistochemical or western blot analysis. A dual-luciferase reporter assay was carried out to validate the regulatory relationship of miR-199a-5p with SOCS7.

Results. The EZH2 level was increased in CC14-treated rats and in TGF- $\beta 1$-treated HSCs, whereas DZNep treatment significantly inhibited EZH2 expression. DZNep repressed hepatic fibrosis in vivo and in vitro, as evidenced by the decrease of hepatic fibrosis markers ( $\alpha$-SMA and Collagen I). Moreover, miR-199a-5p expression was repressed by DZNep in TGF- $\beta 1$-activated HSCs. Notably, downregulation of miR-199a-5p decreased TGF- $\beta 1$-induced expression of fibrosis markers. SOCS7 was identified as a direct target of miR-199a-5p. The expression of SOCS7 was decreased in TGF- $\beta 1$-activated HSCs, but DZNep treatment restored SOCS7 expression. More importantly, SOCS7 knockdown decreased the effect of DZNep on collagen I and $\alpha$-SMA expression in TGF- $\beta 1$-activated HSCs.

Conclusions. DZNep suppresses hepatic fibrosis through regulating miR-199a-5p/SOCS7 axis, suggesting that DZNep may represent a novel treatment for fibrosis.

41 Keywords: Hepatic fibrosis; DZNep; TGF- $\beta 1$; EZH2; miR-199a-5p; SOCS7. 


\section{INTRODUCTION}

Hepatic fibrosis, induced by pro-inflammatory cytokines, chemokines, or viral infection, causes about one million deaths annually worldwide due to the development of cirrhosis (Ezhilarasan et al. 2018; Lee et al. 2015). However, there is currently no effective anti-fibrotic therapy (Bollong et al. 2017). The hepatic stellate cells (HSCs) are the major source of extracellular matrix (ECM) proteins in liver fibrosis, including fibronectin (FN), $\alpha$-smooth muscle actin ( $\alpha$-SMA), and Type I collagen (COL I) (Ellis \& Mann 2012; Pellicoro et al. 2014; Tacke \& Trautwein 2015). Despite commonly progressing to hepatic cirrhosis and organ damage, in principle, hepatic fibrosis is a reversible process. Emerging studies showed that liver fibrosis may be reversed or slowed when hepatic damage is successfully treated (Latasa et al. 2010; Tangkijvanich \& Yee 2002).

The role of the enhancer of zeste homolog 2 (EZH2) in regulating myofibroblast transformation and tissue fibrosis has recently been verified (Xiao et al. 2016). Tsou et al. demonstrated that EZH2 expression is increased in scleroderma dermal fibroblasts and observed that forced expression of EZH2 in fibroblasts results in a pro-fibrotic response, whereas pharmacological inhibition of EZH2 effectively represses scleroderma fibrosis (Tsou et al. 2019). The EZH2 expression is upregulated in atrial fibroblasts, and pharmacological or genetic inhibition of EZH2 represses atrial fibroblast differentiation and results in a decrease in ECM production (Song et al. 2019). The role of EZH2 in regulating liver fibrosis is gradually being elucidated. The EZH2 expression is also elevated in a murine model of liver fibrosis and in TGF- $\beta 1$-activated HSCs (Martin-Mateos et al. 2019). 
Inhibition of EZH2 decreases $\alpha$-SMA expression in TGF- $\beta 1$-activated HSCs by de-repressing DKK-1, an antagonist of Wnt signaling (Yang et al. 2017). Atta et al. showed that the EZH2 expression level is increased in fibrotic liver tissues, but HGF (hepatocyte growth factor) treatment reduces EZH2 expression and represses hepatic fibrosis in rats (Atta et al. 2014). Furthermore, an EZH2 inhibitor, DZNep, has been shown to play an important role in repressing liver fibrosis. Zeybel et al. demonstrated that DZNep presents an anti-fibrotic effect in a murine model of liver fibrosis and in TGF- $\beta 1$-activated HSCs (Zeybel et al. 2017).

MicroRNAs (miRNAs) are a class of small single-stranded and evolutionary conserved noncoding RNA molecules (Ambros 2001) (Eulalio et al. 2008; Liu et al. 2008). Emerging evidence suggests that miRNAs regulate almost all physiological and pathological processes, such as cell differentiation (Ortega et al. 2010), embryonic development (Laurent 2008), tumorigenesis (Kumar et al. 2007), and tissue fibrosis (Patel \& Noureddine 2012). Several functional miRNAs in liver fibrosis have been identified, including miR-29, miR-21, miR-7-5p, miR-33a, and miR129-5p (O'Reilly 2016). Suppressor of cytokine signaling (SOCS) protein family (contains SOCS1-SOCS7) functions as a negative regulator of cytokine receptor signaling (Duncan et al. 2017). SOCSs play an important role in various physiological processes including inflammation, cell viability, and development (Durham et al. 2019; Fu et al. 2020). Emerging studies demonstrated the role of SOCS7 in regulating cancer cells proliferation (Noguchi et al. 2013), or in regulating insulin action (Banks et al. 2005). However, the function of SOCS7 on regulating inflammation and fibrosis remains unclear. 
82

Based on above findings, here we investigated the role of DZNep in regulating hepatic fibrosis and uncovered the mechanism underlying DZNep slowing hepatic fibrosis. We demonstrated that the expression of miR-199a-5p was repressed by DZNep in TGF- $\beta 1$-activated HSCs. Functionally, miR-199a-5p inhibition decreased TGF- $\beta 1$-induced hepatic fibrosis, and knockdown of SOCS7 decreased the effect of DZNep on collagen I and $\alpha$-SMA expression in TGF- $\beta 1$-activated HSCs. These data showed that DZNep contributes to alleviate liver fibrosis through regulating miR-199a$5 \mathrm{p} / \mathrm{SOCS} 7$ axis.

\section{MATERIALS AND METHODS}

\section{Animals and CCl4 and DZNep Treatment}

All experiments in this study were approved by the Animal Ethics Committee of Huashan Hospital, Fudan University (2018 Huashan Hospital JS-030). Male Sprague-Dawley (SD) rats (body weights: 180-200 g) were procured from Trophic Animal Feed High-Tech Co., Ltd. (Jiangsu, China) and kept under the specific-pathogen-free (SPF). CCl4 (20070721) and olive oil (060312) were obtained from Sinopharm Co., Ltd. (Shanghai, China). All rats were randomly divided into 3 groups: the control group ( $\mathrm{n}=5), \mathrm{CCl} 4$-induced group $(\mathrm{n}=5)$ and CCl4-induced + DZNep group $(\mathrm{n}=5)$. The rats in the $\mathrm{CCl}_{4}$-induced group and $\mathrm{CCl}$-induced+DZNep group were injected intraperitoneally (i.p.) with $50 \% \mathrm{CCl}_{4}$ olive oil solution at $1 \mathrm{~mL} / \mathrm{kg}$ body weight two times a week (every Monday and Thursday) for 9 weeks, whereas the rats in the control group were subjected to conventional breeding. Two weeks later, in addition to CCl4, the CCl4-induced+DZNep group 
102

103

104

105

106

107

received DZNep (2 mg/kg, twice per week) by i.p. injection for an additional 6 weeks. Rats were sacrificed with $\mathrm{CO}_{2}$ narcosis, and their livers were subsequently removed, embedded in paraffin, and stained with H\&E and Masson's trichrome. Animals were kept in a colony room at controlled temperature $\left(22^{\circ} \mathrm{C}\right)$, and a $12: 12 \mathrm{~h}$ light-dark cycle, with food and water available. All rats were sacrificed through overetherization and decapitation.

\section{Cell Culture and TGF- $\beta 1$ Treatment}

The human HSC line LX2 (ATCC, Manassas, VA, USA) was cultured in DMEM supplemented with $2 \%$ FBS (HyClone, Logan, UT, USA) at $37^{\circ} \mathrm{C}$ in a humidified atmosphere in $5 \% \mathrm{CO}_{2}$. The cells were treated with $10 \mu \mathrm{g} / \mathrm{L}$ TGF- $\beta 1$ (Peprotech, USA) in DMEM supplemented with 1\% FBS for $48 \mathrm{~h}$. DZNep $(1 \mu \mathrm{mol} / \mathrm{L})$ was added to treat cells for $24 \mathrm{~h}$.

\section{Cell transfection}

MiR-199a-5p mimic, miRNA controls (miRcont), and miR-199a-5p inhibitors were obtained from RIBOBIO (Guangzhou, China). siRNA against SOCS7 (siSOCS7) and negative control (siNC) were obtained from Thermo Fisher (Waltham, MA, USA). All small RNAs (40nM) were transfected into LX2 cells, TGF- $\beta 1$-activated LX2 cells in the presence or absence of DZNep with Lipofectamine $^{\mathrm{TM}} 2000$ (Invitrogen, Carlsbad, CA, USA) following manufacturer's instructions, and then cells were collected to carry out qPCR or western blot analysis after $48 \mathrm{~h}$.

\section{Quantitative real-time PCR (qPCR)}


120 For quantification of miR-199a-5p miRNA expression, cDNA was synthesized with a Reverse

121 Transcription Kit (Thermo Fisher) and quantified with a miRNA RT-PCR Quantitation Kit

122 (Qiagen, Duesseldorf, Germany). For quantification of EZH2, collagen I, $\alpha$-SMA and SOCS7

123 mRNA expression, reverse transcription was performed with a High Capacity cDNA Reverse

124 Transcription Kit (Thermo Fisher), and EZH2, collagen I, $\alpha$-SMA and SOCS7 mRNA levels were

125 measured with SYBR Green Mix (Vazyme, Nanjing, China). All primers employed were the

126 following: miR-199a-5p: Forward: 5'-GCCAAGCCCAGTGTTCAGAC-3', Reverse: 5'-

127 GTGCAGGGTCCGAGGTATTC-3'. $\quad$ EZH2, Forward, 5'-

128 CCCTGACCTCTGTCTTACTTGTGGA-3'; Reverse, 5'-ACGTCAGATGGTGCCAGCAATA-

129 3'; Collagen I, Forward: 5'-CCTCAGGGTATTGCTGGACAAC-3', Reverse, 5'-

130 CAGAAGGACCTTGTTTGCCAGG-3'; $\alpha$-SMA, Forward: 5'- GCGTGGCTATTCCTTCGTTA-

131 3', Reverse, 5'-ATGAAGGATGGCTGGAACAG-3'; $\quad$ SOCS7, Forward: 5'-

132 ACAGGAAGGTTGGGATTCTC-3', Reverse, 5'-CAGCACAGACTCTAACTCTG-3'.

133

134

135

136

137

\section{Western blot analysis}

Equal amounts of total protein (approximate $80 \mu \mathrm{g}$ ) were separated by a 12\% SDS-polyacrylamide gel and transferred onto 0.2- $\mu \mathrm{m}$ PVDF membranes (Roche, Basel, Switzerland). The blots were incubated overnight at $4{ }^{\circ} \mathrm{C}$ with antibodies (anti-EZH2: 1:5000, ab186006, Abcam, CA, USA; anti-Collagen I: 1:1000, ab6308, Abcam; anti- $\alpha-S M A: 1: 1000$, ab19245, CST; anti-SOCS7: 1:1000, ab224589, Abcam; anti- $\beta$-actin: 1:2000, ab8227, Abcam) and were subsequently incubated with a secondary antibody (1:5000, ab205718, Abcam). The protein signals were 
140 detected with ECL (Pierce Biotechnology, Rockford, IL) and digitized and analyzed

141 densitometrically using ImageJ software.

\section{Luciferase reporter assay}

143 The recombinant plasmid of pGL3-SOCS7-3'-UTR (wildtype) or pGL3-SOCS7-3'-UTR-

144 Mutation was constructed by inserting the 3' UTR of SOCS7 or its mutant into the pGL3-promoter

145 vector (Promega). LX2 cells $\left(1 \times 10^{5}\right.$ cells/well) were seeded in a 24 -well plate and cotransfected 146 with miR-199a-5p (40nM) or miRcont, pGL3-SOCS7-3'-UTR (10ng), and pRL-TK (1ng,

147 Promega) using Lipofectamine ${ }^{\mathrm{TM}}$ 2000. After $72 \mathrm{~h}$, luciferase activity was assessed by Dual-

148 Luciferase Assays Kit (Promega) according to the manufacturer's protocol.

149 Immunohistochemistry

150 Tissues were fixed in $4 \%$ paraformaldehyde (PFA) in $4{ }^{\circ} \mathrm{C}$ environments for $24 \mathrm{~h}$. After that step,

151 all tissues were dehydrated in the embedding box and embedded using liquid paraffin according

152 to the manufacturer's instructions (Sigma-Aldrich). The sections (measuring $4 \mu \mathrm{m}$ ) were prepared

153 through a slicer and baked on slide warmers for $30 \mathrm{~min}$. The sections were placed in a wet box, in

154 which a small amount of distilled water was added in combination with $3 \%$ hydrogen peroxide

155 and was incubated at room temperature for $10 \mathrm{~min}$. After washing with PBS and distilled water 3

156 times each and for 3 min each time, goat serum occlusive fluid was added to the wet box to incubate

157 sections for $10 \mathrm{~min}$. Immunohistochemistry was performed by primary antibodies and the

158 corresponding secondary antibody according to instruction procedures. Then, $100 \mu \mathrm{LAB}$ solution

159 (Sigma-Aldrich) was added to each section and restained with hematoxylin for 1-2 min.

Peer] reviewing PDF | (2020:09:53274:2:0:NEW 14 Mar 2021) 
160

161

162

163

164

165

166

167

168

169

170

171

172

173

174

175

176

177

178

\section{Immunofluorescence}

LX2 cells were fixed with 4\% paraformaldehyde for $15 \mathrm{~min}$, followed by washing 3 times with sterile PBS at room temperature (RT). After blocking with 5\% BSA (bovine serum albbumin) in $0.2 \%$ Triton $\mathrm{X}-100$ for $1 \mathrm{~h}$ at RT, slides was incubated with anti-EZH2 primary antibody (1:200; ab191080; Abcam) overnight at $4{ }^{\circ} \mathrm{C}$, followed by incubating with goat anti-rabbit antibody (1:2000; A27039; Invitrogen). After washing 3 times, slides were mounted with glycerin and analyzed using a confocal microscopy (Zeiss LSM710, Jena, Germany). DAPI (Solarbio) was used to visualize the nucleus.

\section{Statistical analysis}

Data are presented as means \pm standard deviation $(\mathrm{SD})$ from at least three independent experiments. The statistical analyses of differences between the two groups were carried out using Student's t-test or one-way analysis of variance (ANOVA) followed by the Scheffé test after evaluating normality of data distribution. SPSS 17.0 (SPSS, Inc., USA) was applied to perform the statistical analysis. $\mathrm{P}<0.05$ was considered to indicate significance.

\section{RESULTS}

\section{DZNep inhibited $\mathrm{CCl}_{4}$-induced liver fibrosis in rats}

To investigate the effects of DZNep on hepatic fibrosis, rats were treated with $\mathrm{CCl} 4$ in the presence or absence of DZNep. As shown in Fig. 1A and B, the results of H\&E and Masson staining 
revealed that the liver tissues of the CCl4-treated group showed extensive necrosis, normal structure destruction and increased collagen deposition. However, DZNep treatment effectively ameliorated CCl4-induced pathologic lesions and collagen deposition. Furthermore, an immunofluorescence analysis was performed to assess the EZH2 protein level and its subcellular localization in TGF- $\beta 1$-activated LX2 cells, and the data showed that EZH2 levels were significantly enhanced in TGF- $\beta 1$-treated LX2 cells compared with control, whereas DZNep treatment inhibited the increase (Fig. 1C). Immunofluorescence analysis also showed that EZH2 was located mainly in the nucleus of HSCs (Fig. 1C).

\section{DZNep suppressed TGF- $\beta 1$-induced LX2 cell activation}

The LX2 cells were treated with TGF- $\beta 1$ in the presence or absence of DZNep, and the antifibrosis effect of DZNep was assessed. Fig. 2A showed that the EZH2 mRNA level was markedly upregulated in TGF- $\beta 1$-activated LX2 cells compared with the control, but DZNep treatment notably repressed the enhancement. Then, collagen I and $\alpha$-SMA expression were evaluated by qPCR and immunoblotting. The qPCR analysis data indicated that collagen I and $\alpha$-SMA mRNA expression was significantly upregulated in TGF- $\beta 1$-activated LX2 cells, whereas DZNep treatment repressed TGF- $\beta 1$-induced upregulation of collagen I and $\alpha$-SMA (Fig. 2B and C). Additionally, immunoblotting was performed to determine protein expression. The protein levels of EZH2, collagen I and $\alpha$-SMA were also markedly upregulated in TGF- $\beta 1$-activated LX2 cells, whereas DZNep treatment inhibited TGF- $\beta 1$-induced upregulation of collagen I and $\alpha$-SMA (Fig. 
198 2D-G). These data demonstrated that DZNep contributed to inhibit the expression of EZH2 and

199 fibrosis markers in vivo and in vitro.

200

201

202

203

204

205

206

207

208

209

210

211

212

213

214

215

216

\section{The effect of miR-199a-5p on fibrosis markers expression in vitro}

We next investigated the mechanism governing the role of DZNep in fibrosis markers expression.

Emerging research has demonstrated that EZH2 epigenetically regulates miRNA expression and that aberrant miRNAs are closely correlated with hepatic fibrosis (Feng et al. 2017; Huang et al. 2019; Kwon et al. 2017). To investigate whether DZNep alleviates fibrosis markers expression by regulating the expression of several miRNA, 13 hepatic fibrosis-related miRNAs (miR-29b, miR34a, miR-34b, miR-15b, miR-16, miR-195, miR-15a, miR-199a, miR-200a, miR-200c, miR-378a, miR-424, and miR-29a) (Jiang et al. 2017) were analyzed using qPCR in TGF- $\beta 1$-activated LX2 cells after DZNep treatment. Fig. 3A showed that miR-199a-5p was the most significantly downregulated miRNA in TGF- $\beta 1$-activated LX2 cells after DZNep treatment. Fig. 3B further showed that the miR-199a-5p level was notably upregulated in LX2 cells after TGF- $\beta 1$ treatment, whereas DZNep treatment inhibited this increase. DZNep also repressed CCl4-induced increase of miR-199a-5p in liver tissues (Supporting Fig. S1A). Next, we investigated the role of miR199a-5p inhibition in fibrosis markers expression. The results from qPCR analysis showed that the miR-199a-5p inhibitor remarkably downregulated miR-199a-5p expression in TGF- $\beta 1$-activated LX2 cells (Fig. 3C). Functionally, miR-199a-5p inhibition repressed TGF- $\beta 1$-induced upregulation of collagen I and $\alpha$-SMA mRNA (Fig. 3D and E). Western blot analysis further 
217 demonstrated that miR-199a-5p inhibition decreased TGF- $\beta 1$-induced protein expression of

218 collagen I and $\alpha$-SMA in LX2 cells (Fig. 3F-H).

\section{SOCS7 was a direct target of miR-199a-5p}

220 To illustrate the role of miR-199a-5p, the potential target genes of miR-199a-5p were screened by

221 prediction websites (PicTar and TargetScan). SOCS7 was identified as one of the candidate targets

222 of miR-199a-5p. To support this statement, recombinant plasmids of pGL3-SOCS7-3'UTR-WT

223 or pGL3-SOCS7-3'UTR-Mut were constructed by inserting SOCS7-3'UTR cDNA or its mutant

224 (SOCS7-3'UTR-Mut) into pGL3 and were cotransfected with miR-199a-5p (Fig. 4A). The

225 luciferase reporter assay demonstrated that miR-199a-5p notably suppressed the luciferase

226 expression of SOCS7-3'UTR-LUC, while the mutation of 5 nucleotides in the 3'UTR of SOCS7

227 resulted in the complete abrogation of the suppressive effect (Fig. 4B and C). Moreover, the

228 SOCS7 mRNA level was notably repressed in LX2 cells after miR-199a-5p mimic treatment (Fig.

229 4D). Western blot analysis further demonstrated that SOCS7 protein expression was inhibited by

230 miR-199a-5p (Fig. 4E and F). Functionally, SOCS7 inhibition resulted in an increased expression

231 of collagen I and $\alpha$-SMA in TGF- $\beta 1$-activated LX2 cells (Supporting Fig. S1B and C).

232 DZNep repressed fibrosis markers expression by upregulating SOCS7

233 Given that DZNep was able to inhibit miR-199a-5p expression in TGF- $\beta 1$-activated LX2 cells and

234 that SOCS7 was a direct target gene of miR-199a-5p, we investigated whether DZNep suppressed

235 fibrosis markers expression by the mediation of SOCS7. To this end, we first assessed whether

236 DZNep regulated SOCS7 expression in TGF- $\beta 1$-activated LX2 cells. Fig. 5A-C showed that the 
237 SOCS7 mRNA and protein levels were decreased in LX2 cells after TGF- $\beta 1$ treatment, whereas 238 DZNep treatment partially inhibited TGF- $\beta 1$-induced downregulation of SOCS7. SOCS7 was 239 inhibited using SOCS7-specific siRNA (siSOCS7) in TGF- $\beta 1$-activated LX2 cells, and then the expression levels of collagen I and $\alpha$-SMA were measured in the presence or absence of DZNep. The expression of SOCS7 was significantly upregulated after DZNep treatment, whereas the effects were attenuated by the treatment with SOCS7-siRNA (Fig. 5D, G and H). More importantly, qPCR and Western blot analysis showed that the mRNA and protein expression of collagen I and $\alpha$-SMA were decreased in the DZNep-treated group, whereas SOCS7 knockdown significantly decreased the role of DZNep in regulating collagen I and $\alpha$-SMA mRNA expression

(Fig. 5E-J). Taken together, these data demonstrated that DZNep could effectively suppress hepatic fibrosis through regulating miR-199a-5p/SOCS7 pathway.

\section{DISCUSSION}

In this study, the effect of DZNep on repressing hepatic fibrosis and its underlying mechanism were further investigated. The current data verified that (i) DZNep alleviated CCl4-induced liver fibrosis in rats and in TGF- $\beta 1$-activated HSCs; (ii) DZNep downregulated the expression of miR199a-5p, and miR-199a-5p inhibition repressed TGF- $\beta 1$-induced fibrosis markers expression; (iii)

SOCS7 was a direct target of miR-199a-5p; and (iv) DZNep repressed fibrosis markers expression by upregulating SOCS7. These results uncovered the crucial role of the DZNep/miR-199a-5p axis 
256 in regulating liver fibrosis by regulating SOCS7 and may provide a therapeutic opportunity for 257 patients with liver fibrosis.

258 Enhancer of zeste homolog 2 (EZH2) is a histone H3 lysine 27 (H3K27)-specific 259 methyltransferase, and EZH2-mediated $\mathrm{H} 3 \mathrm{~K} 27 \mathrm{me} 3$ is a suppressive posttranslational modification 260 (McCabe et al. 2012). Zhang et al. demonstrated that GAS5 recruits EZH2 to the matrix 261 metalloproteinase 9 (MMP9) promoter region, thereby resulting in a subsequent downregulation 262 of MMP9 expression, which relieves renal fibrosis in diabetic nephropathy rats (Zhang et al. 263 2020). EZH2-mediated H3K27 hypermethylation at the cyclooxygenase-2 (COX-2) 264 promoter results in the epigenetic silencing of COX-2, facilitating the fibrotic process in idiopathic 265 pulmonary fibrosis (IPF) (Coward et al. 2014; Mozzetta et al. 2014). The effect of EZH2 on liver 266 fibrosis has also been demonstrated. In murine or cell models of liver fibrosis, EZH2 expression 267 is increased, and upregulated EZH2 inhibits Dickkopf-1 (an antagonist of Wnt/ $\beta$-catenin signaling) to activate Wnt/ $\beta$-catenin signaling and facilitate liver fibrosis (Miao et al. 2013; Yang et al. 2017). Therefore, the inhibition of EZH2 presents an excellent opportunity for regressing liver fibrosis. Martin-Mateos et al. demonstrated that pharmacological (GSK-503) or genetic (small interfering RNA) inhibition of EZH2 results in a significant decrease in TGF- $\beta 1$-induced liver fibrosis markers (Martin-Mateos et al. 2019). Carnosol, a compound extracted from rosemary, also shows an antifibrotic effect by repressing EZH2 (Zhao et al. 2018). Several small molecular inhibitors of EZH2, such as EPZ-6438, GSK126, and DZNep, have exhibited therapeutic potential in human diseases, including tumor and tissue fibrosis (Zeybel et al. 2017). In particular, the role of DZNep 
in inhibiting hepatic fibrosis progression has been verified. Zeybel et al. demonstrated that DZNep

277

278

279

280

281

282

283

284

285

286

287

288

289

290

291

292

293

294

295

treatment is effective in repressing hepatic fibrosis progression by selectively targeting HSCderived myofibroblasts (Zeybel et al. 2017). In this study, we verified that DZNep treatment effectively alleviated hepatic fibrosis progression in vitro and in vivo, but we further explored the specific mechanism by which DZNep alleviates liver fibrosis. Our data uncovered the specific mechanism of DZNep in the repression of fibrosis markers expression by regulating miR-199a$5 \mathrm{p} / \mathrm{SOCS} 7$ axis.

Previous studies identified that increasing miR-200a level could alleviate liver fibrosis. Hu et al. demonstrated that IL-22 inhibits HSC activation and ameliorates liver fibrosis through enhancing expression of miR-200a and reducing expression of $\beta$-catenin (Hu et al. 2016). Sun et al. showed that miR-200a has an alleviative effect on HSC activation by decreasing the expression of $\beta$ catenin and TGF- $\beta 2$ (Sun et al. 2014). However, the results of this study indicated that the expression of miR-200a did not change significantly by DZNep treatment. Our data indicated that DZNep treatment inhibited the expression of miR-199a-5p, and downregulated miR-199a-5p contributed to repress TGF- $\beta 1$-induced expression of fibrosis markers. The pulmonary miR-199a$5 p$ level is upregulated in IPF patients and regulates TGF- $\beta$-induced lung fibroblast activation (Lino Cardenas et al. 2013). However, several studies have shown that exosomal miR-199a-5p suppresses the fibrogenic response by inhibiting connective tissue growth factor (CCN2) in HSCs (Chen et al. 2016). These studies discovered that the miR-199a-5p level is higher in quiescent HSC-derived exosomes than in activated HSC-derived exosomes, and exosomal miR-199a-5p is 
296

297

298

299

300

301

302

303

304

305

306

307

308

309

310

311

312

313

314

transferred from quiescent HSCs to activated HSCs and thus represses HSCs fibrosis. In this study, the miR-199a-5p level was markedly increased in LX2 cells after TGF- $\beta 1$ treatment, whereas

DZNep treatment repressed the increase. We next explored the role of miR-199a-5p inhibition in fibrosis markers expression, and our results indicated that downregulation of miR-199a-5p reversed TGF- $\beta 1$-induced upregulation of collagen I and $\alpha$-SMA. SOCS7 was identified as a novel target gene of miR-199a-5p. SOCS7 expression was decreased in TGF- $\beta 1$-activated HSCs, but DZNep treatment restored SOCS7 expression. Importantly, SOCS7 inhibition weakened the effect of DZNep on collagen I and $\alpha$-SMA expression in TGF- $\beta 1$-activated HSCs.

\section{CONCLUSIONS}

The current study demonstrate that DZNep presents an antifibrotic effect by regulating the miR199a-5p/SOCS7 pathway, and further study is essential to investigating whether DZNep can be applied as a therapeutic target for fibrosis.

\section{ETHICS APPROVAL AND CONSENT TO PARTICIPATE}

All experiments in this study were approved by the Animal Ethics Committee of Huashan Hospital, Fudan University (2018 Huashan Hospital JS-030).

\section{DATA AVAILABILTY}

The following information was supplied regarding data availability: The raw data has been supplied as a Supplementary File. 


\section{AUTHOR CONTRIBUTIONS}

316 All authors conceived and designed the experiments, performed the experiments, analyzed the

317 data, prepared the figures and/or tables and drafted the work or revised it critically for important

318 content.

\section{ACKNOWLEDGEMENTS}

320 This work was mainly supported by the National Natural Science Foundation of China (award

321 number 81371821) and the Natural Science Foundation Project of Shanghai (NO. 19ZR1407800).

322 All sponsors or funders played no roles in the study design, data collection and analysis, decision

323 to publish, or preparation of the manuscript.

\section{CONFLICT OF INTEREST}

325 The authors declare that they have no competing interests.

326

327

328

329

330

331

332

333

\section{LIST OF ABBREVIATIONS}

DZNep, 3-deazaneplanocin A; HSCs, hepatic stellate cells; EZH2, enhancer of zeste homolog 2;

SOCS7, suppressor of cytokine signaling 7; $\alpha$-SMA, Alpha-smooth muscle actin; Collagen I, type I collagen; TGF- $\beta 1$, transforming growth factor- $\beta 1 ; \mathrm{CCl}_{4}$, carbon tetrachloride; ECM, Extracellular matrix; H\&E, Hematoxylin and Eosin; DKK-1, dickkopf WNT signaling pathway inhibitor 1; NF- $\mathrm{B}$, nuclear factor kappa B; ATCC, American Type Culture Collection; FBS, fetal bovine serum; DMEM, Dulbecco Modified Eagle Medium; NC, negative controls; SDS, Sodium Dodecyl

Sulfonate; ECL, chemiluminescence; PFA, paraformaldehyde; DAB, 3,3'-diaminobenzidine; 
334 PBS, phosphate buffer saline; SD, standard deviation; ANOVA, one-way analysis of variance;

335

336

qPCR, quantitative real-time Polymerase Chain Reaction; H3K27, histone H3 lysine 27; MMP9,

matrix metalloproteinase 9; COX-2, cyclooxygenase-2.

\section{PATIENT CONSENT FOR PUBLICATION}

Not applicable.

\section{REFERENCES}

Ambros V. 2001. microRNAs: tiny regulators with great potential. Cell 107:823-826.

Atta H, El-Rehany M, Hammam O, Abdel-Ghany H, Ramzy M, Roderfeld M, Roeb E, Al-Hendy A, Raheim SA, Allam H, and Marey H. 2014. Mutant MMP-9 and HGF gene transfer enhance resolution of CCl4-induced liver fibrosis in rats: role of ASH1 and EZH2 methyltransferases repression. PLoS One 9:e112384.

Banks AS, Li J, McKeag L, Hribal ML, Kashiwada M, Accili D, and Rothman PB. 2005. Deletion of SOCS7 leads to enhanced insulin action and enlarged islets of Langerhans. $J$ Clin Invest 115:2462-2471.

Bollong MJ, Yang B, Vergani N, Beyer BA, Chin EN, Zambaldo C, Wang D, Chatterjee AK, Lairson LL, and Schultz PG. 2017. Small molecule-mediated inhibition of myofibroblast transdifferentiation for the treatment of fibrosis. Proc Natl Acad Sci U S A 114:4679-4684.

Chen L, Chen R, Velazquez VM, and Brigstock DR. 2016. Fibrogenic Signaling Is Suppressed in Hepatic Stellate Cells through Targeting of Connective Tissue Growth Factor (CCN2) by Cellular or Exosomal MicroRNA199a-5p. Am J Pathol 186:2921-2933.

Coward WR, Feghali-Bostwick CA, Jenkins G, Knox AJ, and Pang L. 2014. A central role for G9a and EZH2 in the epigenetic silencing of cyclooxygenase-2 in idiopathic pulmonary fibrosis. FASEB J 28:3183-3196.

Duncan SA, Baganizi DR, Sahu R, Singh SR, and Dennis VA. 2017. SOCS Proteins as Regulators of Inflammatory Responses Induced by Bacterial Infections: A Review. Front Microbiol 8:2431.

Durham GA, Williams JJL, Nasim MT, and Palmer TM. 2019. Targeting SOCS Proteins to Control JAK-STAT Signalling in Disease. Trends Pharmacol Sci 40:298-308.

Ellis EL, and Mann DA. 2012. Clinical evidence for the regression of liver fibrosis. J Hepatol 56:1171-1180.

Eulalio A, Huntzinger E, and Izaurralde E. 2008. Getting to the root of miRNA-mediated gene silencing. Cell 132:914.

Ezhilarasan D, Sokal E, and Najimi M. 2018. Hepatic fibrosis: It is time to go with hepatic stellate cell-specific therapeutic targets. Hepatobiliary Pancreat Dis Int 17:192-197.

Peer] reviewing PDF | (2020:09:53274:2:0:NEW 14 Mar 2021) 
Feng GX, Li J, Yang Z, Zhang SQ, Liu YX, Zhang WY, Ye LH, and Zhang XD. 2017. Hepatitis B virus X protein promotes the development of liver fibrosis and hepatoma through downregulation of miR-30e targeting P4HA2 mRNA. Oncogene 36:6895-6905.

Fu B, Yin S, Lin X, Shi L, Wang Y, Zhang S, Zhao Q, Li Z, Yang Y, and Wu H. 2020. PTPN14 aggravates inflammation through promoting proteasomal degradation of SOCS7 in acute liver failure. Cell Death Dis 11:803.

Hu BL, Shi C, Lei RE, Lu DH, Luo W, Qin SY, Zhou Y, and Jiang HX. 2016. Interleukin-22 ameliorates liver fibrosis through miR-200a/beta-catenin. Sci Rep 6:36436.

Huang YH, Kuo HC, Yang YL, and Wang FS. 2019. MicroRNA-29a is a key regulon that regulates BRD4 and mitigates liver fibrosis in mice by inhibiting hepatic stellate cell activation. Int J Med Sci 16:212-220.

Jiang XP, Ai WB, Wan LY, Zhang YQ, and Wu JF. 2017. The roles of microRNA families in hepatic fibrosis. Cell Biosci 7:34.

Kumar MS, Lu J, Mercer KL, Golub TR, and Jacks T. 2007. Impaired microRNA processing enhances cellular transformation and tumorigenesis. Nat Genet 39:673-677.

Kwon H, Song K, Han C, Zhang J, Lu L, Chen W, and Wu T. 2017. Epigenetic Silencing of miRNA-34a in Human Cholangiocarcinoma via EZH2 and DNA Methylation: Impact on Regulation of Notch Pathway. Am J Pathol 187:2288-2299.

Latasa MU, Gil-Puig C, Fernandez-Barrena MG, Rodriguez-Ortigosa CM, Banales JM, Urtasun R, Goni S, Mendez M, Arcelus S, Juanarena N, Recio JA, Lotersztajn S, Prieto J, Berasain C, Corrales FJ, Lecanda J, and Avila MA. 2010. Oral methylthioadenosine administration attenuates fibrosis and chronic liver disease progression in Mdr2-/- mice. PLoS One 5:e15690.

Laurent LC. 2008. MicroRNAs in embryonic stem cells and early embryonic development. J Cell Mol Med 12:21812188.

Lee YA, Wallace MC, and Friedman SL. 2015. Pathobiology of liver fibrosis: a translational success story. Gut 64:830-841.

Lino Cardenas CL, Henaoui IS, Courcot E, Roderburg C, Cauffiez C, Aubert S, Copin MC, Wallaert B, Glowacki F, Dewaeles E, Milosevic J, Maurizio J, Tedrow J, Marcet B, Lo-Guidice JM, Kaminski N, Barbry P, Luedde T, Perrais M, Mari B, and Pottier N. 2013. miR-199a-5p Is upregulated during fibrogenic response to tissue injury and mediates TGFbeta-induced lung fibroblast activation by targeting caveolin-1. PLoS Genet 9:e1003291.

Liu X, Fortin K, and Mourelatos Z. 2008. MicroRNAs: biogenesis and molecular functions. Brain Pathol 18:113-121. Martin-Mateos R, De Assuncao TM, Arab JP, Jalan-Sakrikar N, Yaqoob U, Greuter T, Verma VK, Mathison AJ, Cao S, Lomberk G, Mathurin P, Urrutia R, Huebert RC, and Shah VH. 2019. Enhancer of Zeste Homologue 2 Inhibition Attenuates TGF-beta Dependent Hepatic Stellate Cell Activation and Liver Fibrosis. Cell Mol Gastroenterol Hepatol 7:197-209.

McCabe MT, Ott HM, Ganji G, Korenchuk S, Thompson C, Van Aller GS, Liu Y, Graves AP, Della Pietra A, 3rd, Diaz E, LaFrance LV, Mellinger M, Duquenne C, Tian X, Kruger RG, McHugh CF, Brandt M, Miller WH, Dhanak D, Verma SK, Tummino PJ, and Creasy CL. 2012. EZH2 inhibition as a therapeutic strategy for lymphoma with EZH2-activating mutations. Nature 492:108-112.

Miao CG, Yang YY, He X, Huang C, Huang Y, Zhang L, Lv XW, Jin Y, and Li J. 2013. Wnt signaling in liver fibrosis: progress, challenges and potential directions. Biochimie 95:2326-2335.

Peer] reviewing PDF | (2020:09:53274:2:0:NEW 14 Mar 2021) 
Mozzetta C, Pontis J, Fritsch L, Robin P, Portoso M, Proux C, Margueron R, and Ait-Si-Ali S. 2014. The histone H3 lysine 9 methyltransferases G9a and GLP regulate polycomb repressive complex 2-mediated gene silencing. Mol Cell 53:277-289.

Noguchi S, Yamada N, Kumazaki M, Yasui Y, Iwasaki J, Naito S, and Akao Y. 2013. socs7, a target gene of microRNA-145, regulates interferon-beta induction through STAT3 nuclear translocation in bladder cancer cells. Cell Death Dis 4:e482.

O'Reilly S. 2016. MicroRNAs in fibrosis: opportunities and challenges. Arthritis Res Ther 18:11.

Ortega FJ, Moreno-Navarrete JM, Pardo G, Sabater M, Hummel M, Ferrer A, Rodriguez-Hermosa JI, Ruiz B, Ricart W, Peral B, and Fernandez-Real JM. 2010. MiRNA expression profile of human subcutaneous adipose and during adipocyte differentiation. PLoS One 5:e9022.

Patel V, and Noureddine L. 2012. MicroRNAs and fibrosis. Curr Opin Nephrol Hypertens 21:410-416.

Pellicoro A, Ramachandran P, Iredale JP, and Fallowfield JA. 2014. Liver fibrosis and repair: immune regulation of wound healing in a solid organ. Nat Rev Immunol 14:181-194.

Song S, Zhang R, Mo B, Chen L, Liu L, Yu Y, Cao W, Fang G, Wan Y, Gu Y, Wang Y, Li Y, and Wang Q. 2019. EZH2 as a novel therapeutic target for atrial fibrosis and atrial fibrillation. $J$ Mol Cell Cardiol 135:119-133.

Sun X, He Y, Ma TT, Huang C, Zhang L, and Li J. 2014. Participation of miR-200a in TGF-beta1-mediated hepatic stellate cell activation. Mol Cell Biochem 388:11-23.

Tacke F, and Trautwein C. 2015. Mechanisms of liver fibrosis resolution. J Hepatol 63:1038-1039.

Tangkijvanich P, and Yee HF, Jr. 2002. Cirrhosis--can we reverse hepatic fibrosis? Eur J Surg Suppl:100-112.

Tsou PS, Campbell P, Amin MA, Coit P, Miller S, Fox DA, Khanna D, and Sawalha AH. 2019. Inhibition of EZH2 prevents fibrosis and restores normal angiogenesis in scleroderma. Proc Natl Acad Sci U S A 116:3695-3702.

Xiao X, Senavirathna LK, Gou X, Huang C, Liang Y, and Liu L. 2016. EZH2 enhances the differentiation of fibroblasts into myofibroblasts in idiopathic pulmonary fibrosis. Physiol Rep 4.

Yang Y, Chen XX, Li WX, Wu XQ, Huang C, Xie J, Zhao YX, Meng XM, and Li J. 2017. EZH2-mediated repression of Dkk1 promotes hepatic stellate cell activation and hepatic fibrosis. $J$ Cell Mol Med 21:2317-2328.

Zeybel M, Luli S, Sabater L, Hardy T, Oakley F, Leslie J, Page A, Moran Salvador E, Sharkey V, Tsukamoto H, Chu DCK, Singh US, Ponzoni M, Perri P, Di Paolo D, Mendivil EJ, Mann J, and Mann DA. 2017. A Proof-ofConcept for Epigenetic Therapy of Tissue Fibrosis: Inhibition of Liver Fibrosis Progression by 3Deazaneplanocin A. Mol Ther 25:218-231.

Zhang L, Zhao S, and Zhu Y. 2020. Long noncoding RNA growth arrest-specific transcript 5 alleviates renal fibrosis in diabetic nephropathy by downregulating matrix metalloproteinase 9 through recruitment of enhancer of zeste homolog 2. FASEB J 34:2703-2714.

Zhao H, Wang Z, Tang F, Zhao Y, Feng D, Li Y, Hu Y, Wang C, Zhou J, Tian X, and Yao J. 2018. Carnosol-mediated Sirtuin 1 activation inhibits Enhancer of Zeste Homolog 2 to attenuate liver fibrosis. Pharmacol Res 128:327337.

\section{FIGURE LEGENDS}

Peer) reviewing PDF | (2020:09:53274:2:0:NEW 14 Mar 2021) 

assessed using H\&E (A) and Masson (B) staining in the liver tissues of each group of rats $(n=5)$. (C) LX2 cells were treated with TGF- $\beta 1(10 \mu \mathrm{g} / \mathrm{L})$ in the presene or absence of DZNep $(1 \mu \mathrm{mol} / \mathrm{L})$, and then the expression and subcellular localization of EZH2 in LX2 cells was assessed using immunofluorescence analysis. Scan bar, $20 \mu \mathrm{m}$.

\section{Figure 2. DZNep suppressed TGF- $\beta 1$-induced LX2 cell fibrosis}

The LX2 cells were treated with TGF- $\beta 1(10 \mu \mathrm{g} / \mathrm{L})$ for $48 \mathrm{~h}$ in the presence or absence of DZNep

( $1 \mu \mathrm{mol} / \mathrm{L})$ for $24 \mathrm{~h}$. The cell model was divided into three groups: 1) Control group; 2$)$ TGF- $\beta 1$ -

activated group; and 3) TGF- $\beta 1$-activated+DZNep group. (A-C) qPCR analysis of EZH2 (A), collagen I (B) and $\alpha$-SMA (C) mRNA expression in each group of cells. (D-G) Representative western blot of EZH2 (D and E), collagen I (D and F), $\alpha$-SMA (D and G) protein expression in each group of cells, and quantification of average across three separate experiments. ${ }^{*} \mathrm{P}<0.05$, $* * \mathrm{P}<0.01$.

(A) The expression level of 13 hepatic fibrosis-related miRNAs (miR-29b, miR-34a, miR-34b, miR-15b, miR-16, miR-195, miR-15a, miR-199a, miR-200a, miR-200c, miR-378a, miR-424 and miR-29a) was assessed using qPCR analysis in TGF- $\beta 1$-activated LX2 cells in the presence or 
462 absence of DZNep treatment $(n=3)$. (B) The expression level of miR-199a-5p was assessed using 463 qPCR analysis in TGF- $\beta 1$-activated LX2 cells in the presence or absence of DZNep treatment. (C-

464 E) The expression levels of miR-199a-5p (C), collagen I (D) and $\alpha$-SMA (E) were assesed using 465 qPCR analysis in LX2 control cells compared with TGF- $\beta 1$-activated LX2 cells treated with 466 miRcont inhibitor or miR-199a-5p inhibitor. (F-H) Representative western blot of collagen I (F 467 and $\mathrm{G}$ ) and $\alpha-\mathrm{SMA}(\mathrm{F}$ and $\mathrm{H})$ protein expression in TGF- $\beta 1$-activated LX2 cells treated with NC 468 inhibitor or miR-199a-5p inhibitor, and quantification of average across three separate 469 experiments. $* \mathrm{P}<0.05, * * \mathrm{P}<0.01$.

Figure 4. SOCS7 was a direct target of miR-199a-5p

471

472

473

(A) Schematic representation of the miR-199a-5p site in SOCS7-3'UTR. (B) The expression of miR-199a-5p was assessed using qPCR analysis in LX2 cells transfected with miRcont inhibitor or miR-199a-5p mimic. (C) Luciferase activity was assayed in LX2 cells cotransfected with miR199a-5p and luciferase reporters containing SOCS7-3'UTR. Data are presented as the relative ratio of firefly luciferase activity to Renilla luciferase activity. (D) The mRNA expression of SOCS7 was assessed using qPCR analysis in LX2 cells transfected with NC or miR-199a-5p mimic. (E and F) Representative western blot of SOCS7 protein expression in LX2 cells transfected with miRcont or miR-199a-5p mimic, and quantification of average across three separate experiments. $* \mathrm{P}<0.05,{ }^{* * *} \mathrm{P}<0.001$.

\section{Figure 5. DZNep repressed liver fibrosis by upregulating SOCS7}


481 (A) The mRNA expression of SOCS7 was detected by qPCR analysis in TGF- $\beta 1$-activated LX2 482 cells in the presence or absence of DZNep treatment. (B and C) Western blot and 483 quantitative analysis of SOCS7 protein expression in TGF- $\beta 1$-activated LX2 cells in the presence 484 or absence of DZNep treatment. (D-F) The mRNA expression of SOCS7 (D), collagen I (E) and 485 $\alpha$-SMA (F) was detected by qPCR analysis in TGF- $\beta 1$-activated LX2 cells in the presence or 486 absence of DZNep treatment after SOCS7 knockdown with siRNA-NC or SOCS7. (G-J) Representative Western blot of SOCS7 ( $\mathrm{G}$ and H), collagen I ( $\mathrm{G}$ and I) and $\alpha$-SMA $(\mathrm{G}$ and J) in DZNep-treated LX2 cells in the presence of siRNA-NC (siNC) or siSOCS7, and quantification of average across three separate experiments. ${ }^{*} \mathrm{P}<0.05, * * \mathrm{P}<0.01$.

Supporting Figure S1. (A) The expression level of miR-199a-5p was assessed using qPCR analysis in the liver tissues of control group, $\mathrm{CCl} 4$ group, and CCl4+DZNep group. The mRNA treatment with siNC or siSOCS7. $* * \mathrm{P}<0.01$. 
Figure 1

DZNep resisted $\mathrm{CCl}_{4}$-induced liver fibrosis in rats

The rat model was divided into three groups: 1) Control group; 2) $\mathrm{CCl}_{4}$-induced group; and 3) $\mathrm{CCl}_{4}$-induced+DZNep group. (A-B) The hepatic pathologic lesions and collagen deposition were assessed using $H \& E(A)$ and Masson (B) staining in the liver tissues of each group of rats $(n=5)$. (C) LX2 cells were treated with TGF- $\beta 1(10 \mu \mathrm{g} / \mathrm{L})$ in the presene or absence of DZNep ( $1 \mu \mathrm{mol} / \mathrm{L})$, and then the expression and subcellular localization of EZH2 in LX2 cells was assessed using immunofluorescence analysis. Scan bar, $20 \mu m$. 
A

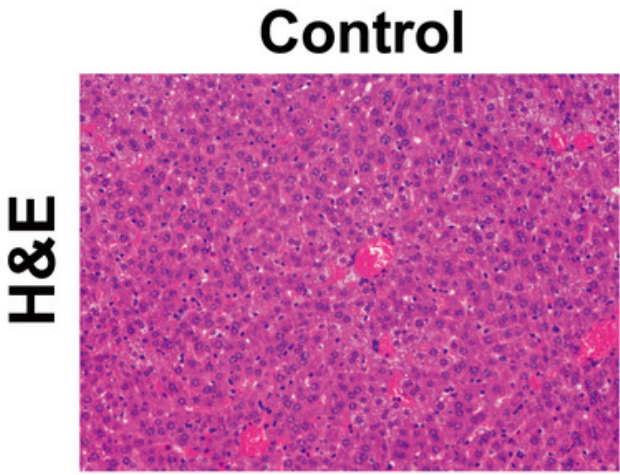

B

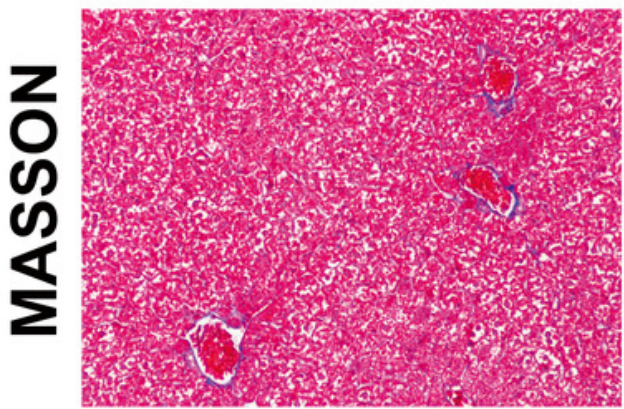

C

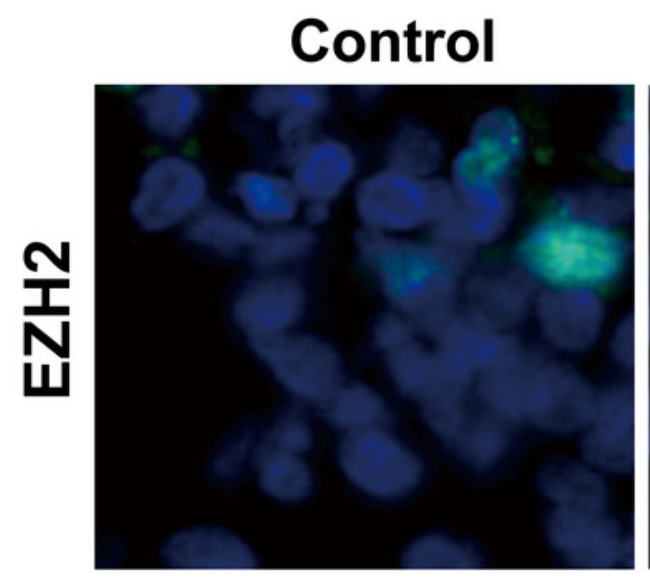

$\mathrm{CCl} 4$
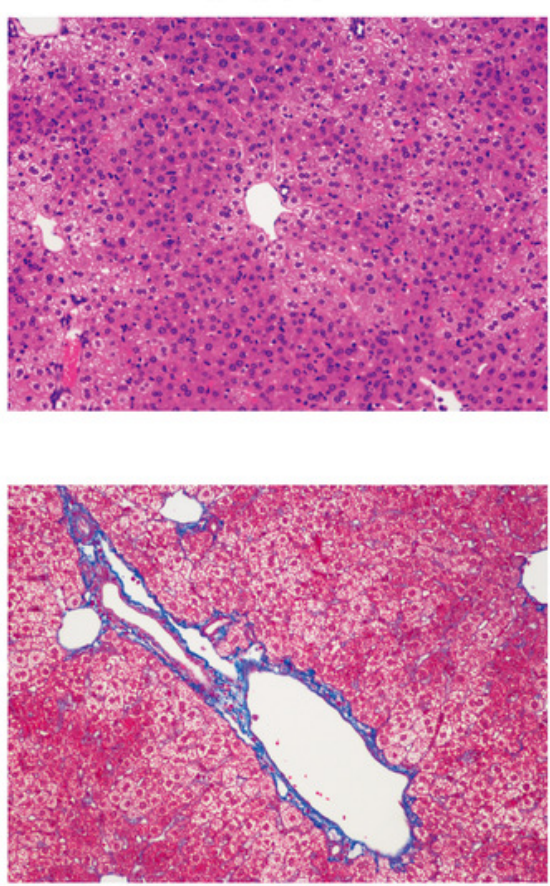

TGF- $\beta 1$

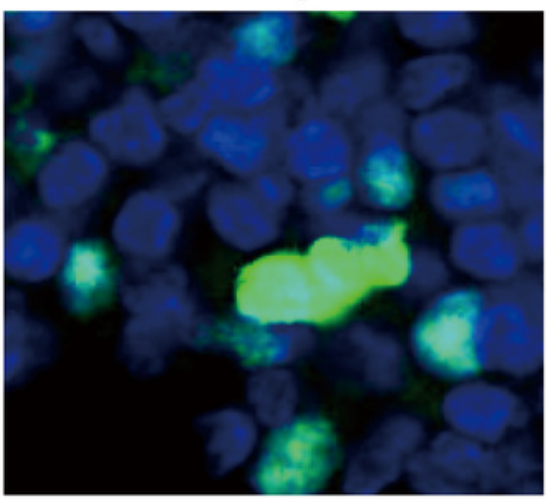

CCl4+DZNep
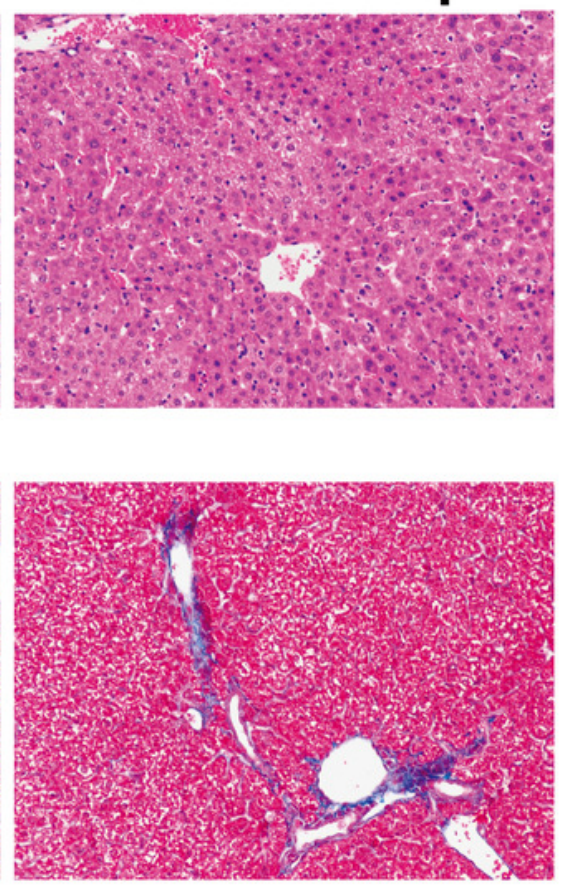

TGF- $\beta 1+D Z N e p$

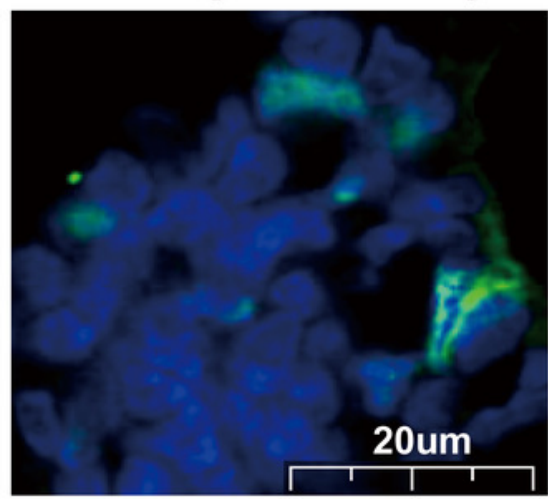




\section{Figure 2}

DZNep suppressed TGF- $\beta 1$-induced LX2 cell fibrosis

The LX2 cells were treated with TGF- $\beta 1(10 \mu \mathrm{g} / \mathrm{L})$ for $48 \mathrm{~h}$ in the presence or absence of DZNep ( $1 \mu \mathrm{mol} / \mathrm{L}$ ) for $24 \mathrm{~h}$. The cell model was divided into three groups: 1 ) Control group; 2 ) TGF- $\beta 1$-activated group; and 3) TGF- $\beta 1$-activated+DZNep group. (A-C) qPCR analysis of EZH2 (A), collagen I (B) and $\alpha \square S M A(C)$ mRNA expression in each group of cells. (D-G)

Representative western blot of EZH2 ( $D$ and $E$ ), collagen I ( $D$ and $F$ ), $\alpha \square S M A$ ( $D$ and $G$ ) protein expression in each group of cells, and quantification of average across three separate experiments. $* \mathrm{P}<0.05, * * \mathrm{P}<0.01$.

A
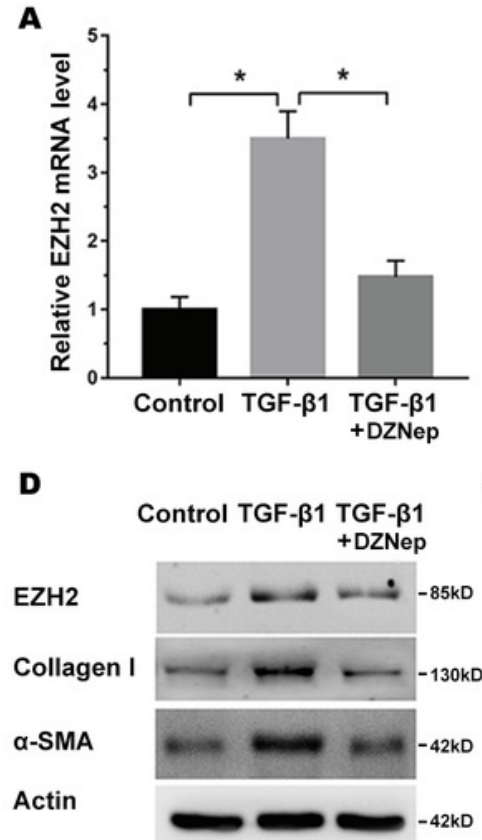

B
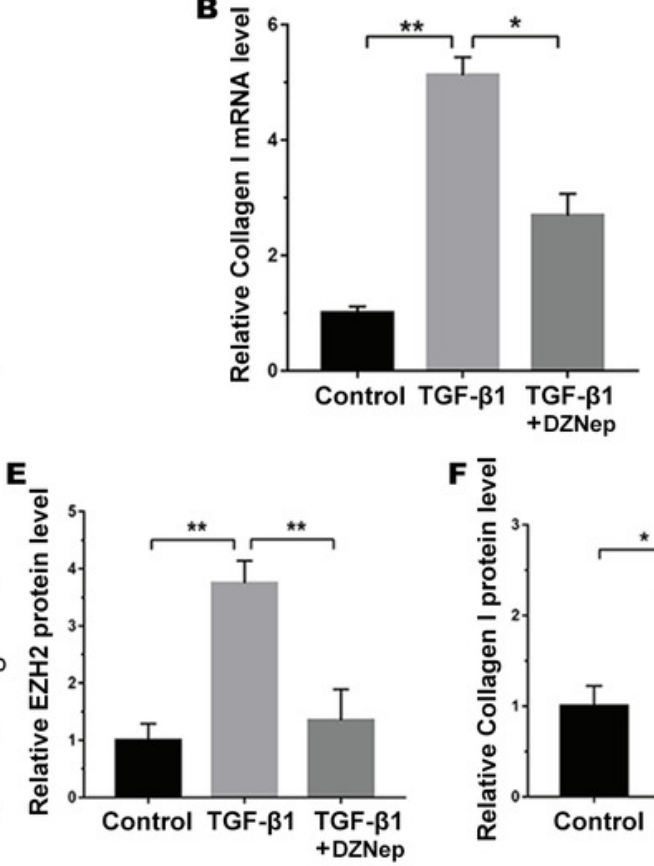

C
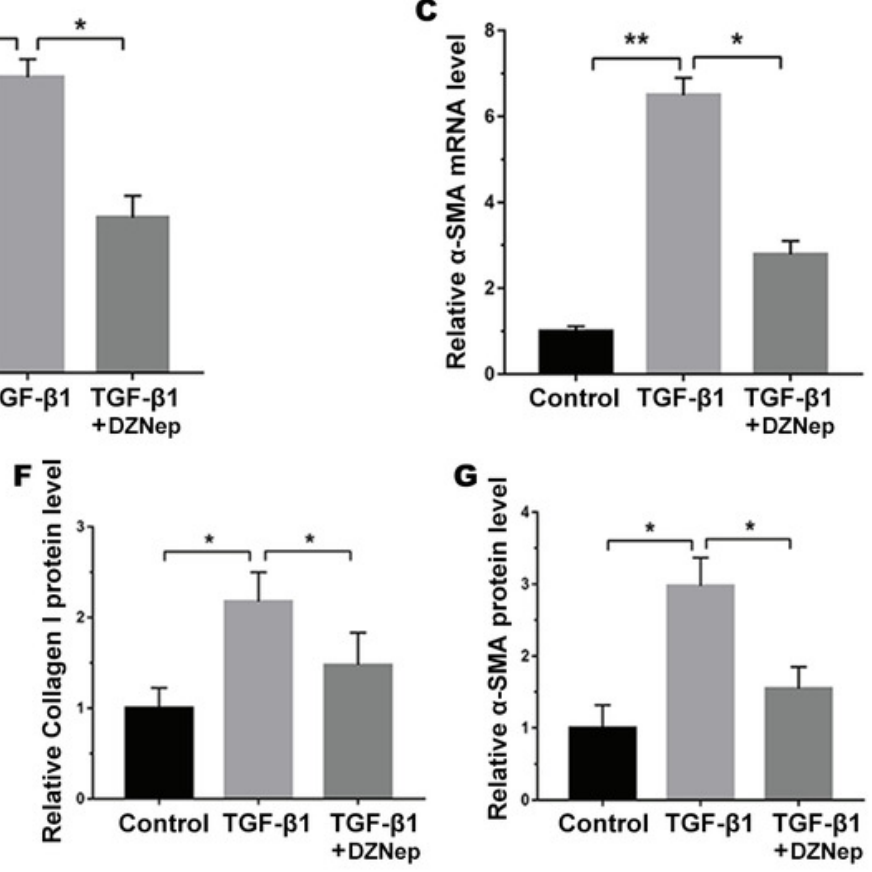


\section{Figure 3}

The effect of miR-199a-5p on hepatic fibrosis in vitro

(A) The expression level of 13 hepatic fibrosis-related miRNAs (miR-29b, miR-34a, miR-34b, miR-15b, miR-16, miR-195, miR-15a, miR-199a, miR-200a, miR-200c, miR-378a, miR-424 and miR-29a) was assessed using qPCR analysis in TGF- $\beta 1$-activated LX2 cells in the presence or absence of DZNep treatment ( $n=3)$. (B) The expression level of miR-199a-5p was assessed using qPCR analysis in TGF- $\beta 1$-activated LX2 cells in the presence or absence of DZNep treatment. (C-E) The expression levels of miR-199a-5p (C), collagen I (D) and $\alpha \square S M A(E)$ were assesed using qPCR analysis in LX2 control cells compared with TGF- $\beta 1$-activated LX2 cells treated with miRcont inhibitor or miR-199a-5p inhibitor. (F-H) Representative western blot of collagen I ( $F$ and $\mathrm{G}$ ) and $\alpha \square S M A(F$ and $H$ ) protein expression in TGF- $\beta 1$-activated LX2 cells treated with NC inhibitor or miR-199a-5p inhibitor, and quantification of average across three separate experiments. $* \mathrm{P}<0.05, * * \mathrm{P}<0.01$.

A

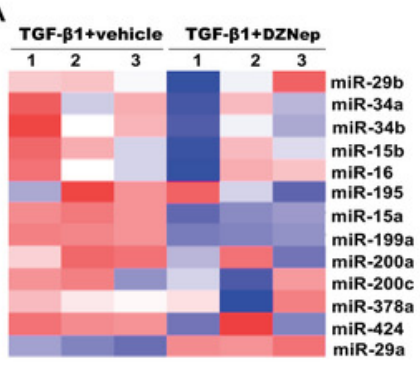

E

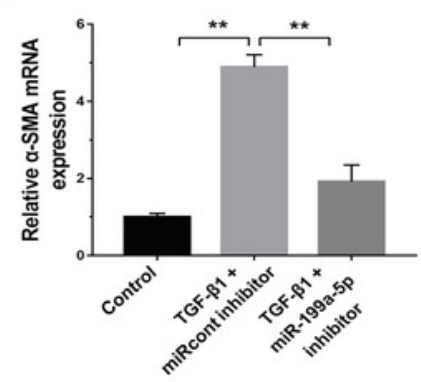

B
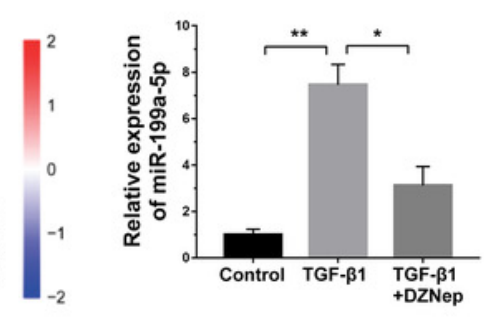

$\mathbf{F}$

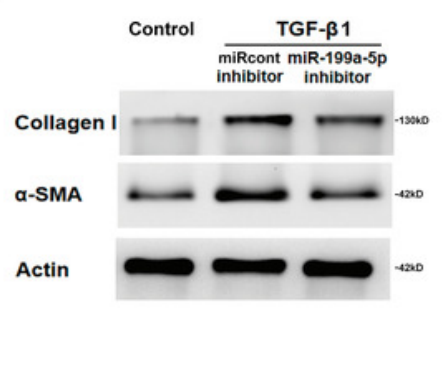

C

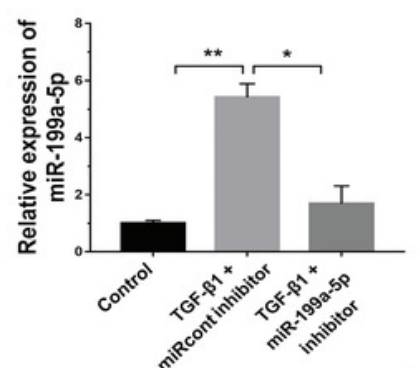

G

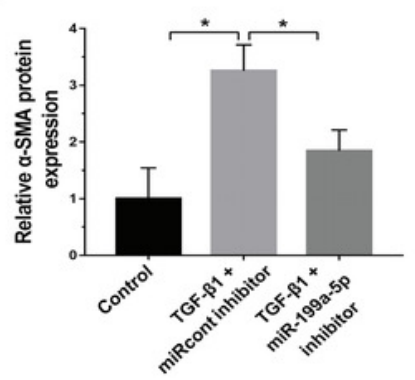

D

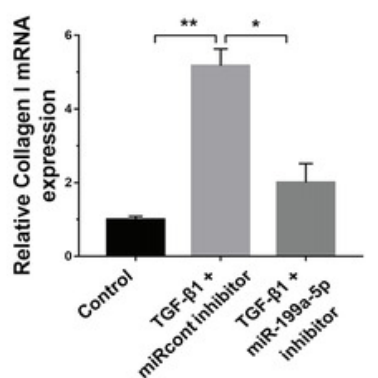

H

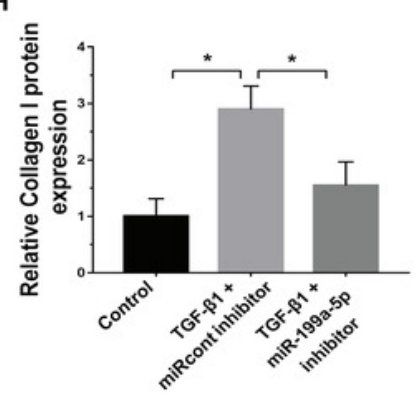




\section{Figure 4}

SOCS7 was a direct target of miR-199a-5p

(A) Schematic representation of the miR-199a-5p site in SOCS7-3'UTR. (B) The expression of miR-199a-5p was assessed using qPCR analysis in LX2 cells transfected with miRcont inhibitor or miR-199a-5p mimic. (C) Luciferase activity was assayed in LX2 cells cotransfected with miR-199a-5p and luciferase reporters containing SOCS7-3'UTR. Data are presented as the relative ratio of firefly luciferase activity to Renilla luciferase activity. (D) The mRNA expression of SOCS7 was assessed using 9PCR analysis in LX2 cells transfected with NC or miR-199a-5p mimic. (E and F) Representative western blot of SOCS7 protein expression in LX2 cells transfected with miRcont or miR-199a-5p mimic, and quantification of average across three separate experiments. $* \mathrm{P}<0.05, * * * \mathrm{P}<0.001$. 
A

SOCS7 3' UTR WT 5'...GCUGUUGGAAUGCCCACACUGGU.. hsa-miR-199a-5p 3' CUUGUCCAUCAGACUUGUGACCC socs7 3' UTR Mut 5'...GCUGUUGgAAUgCCCGAAAUAA

C

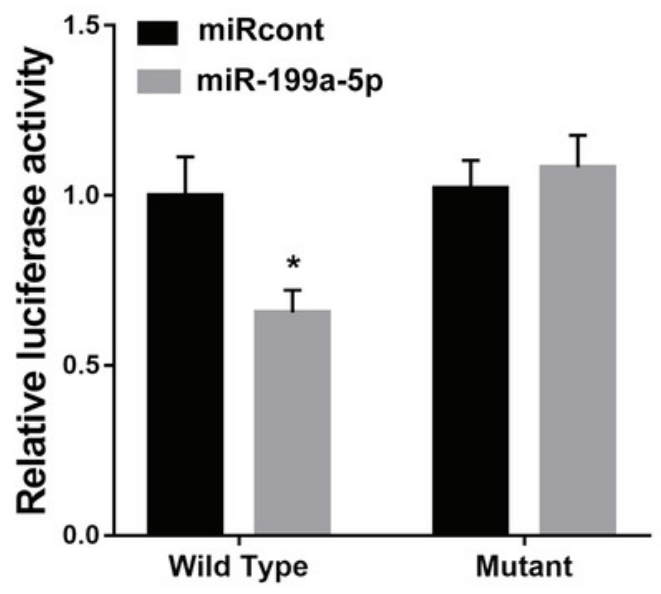

E

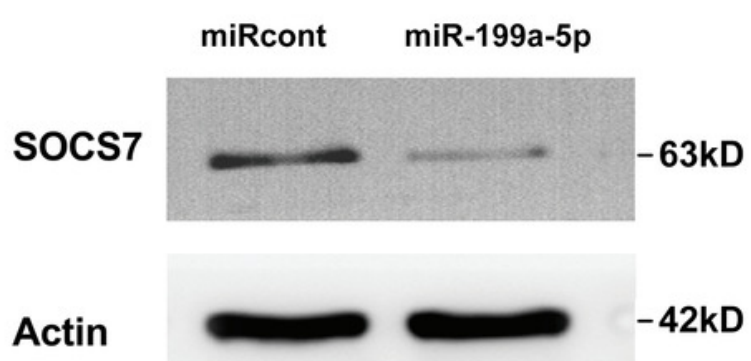

B

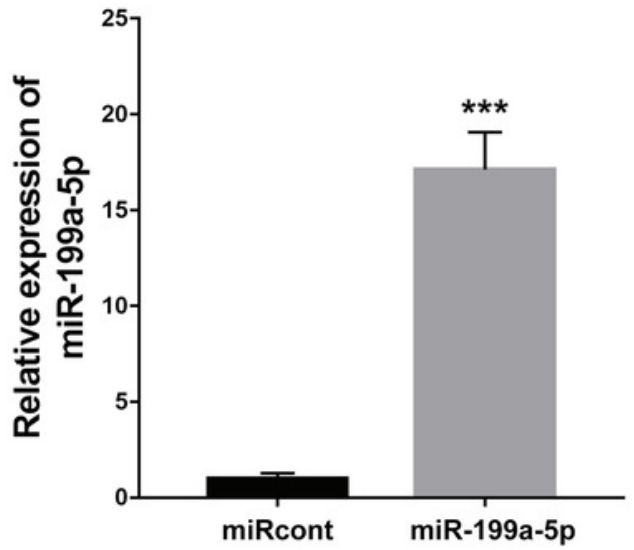

D

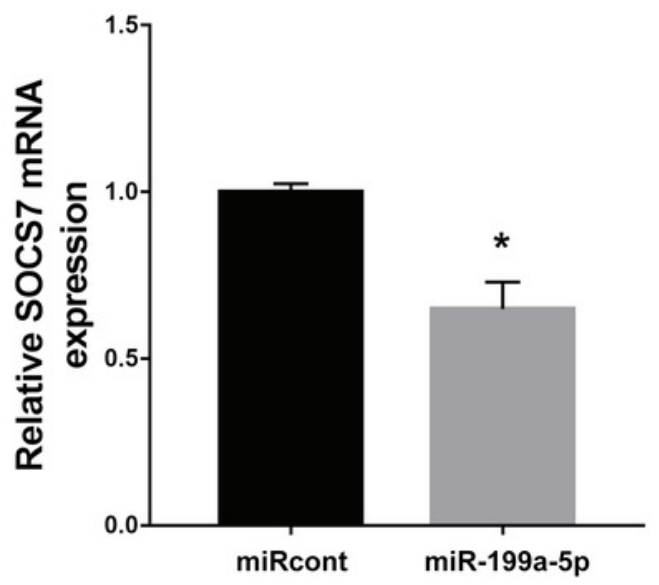

F

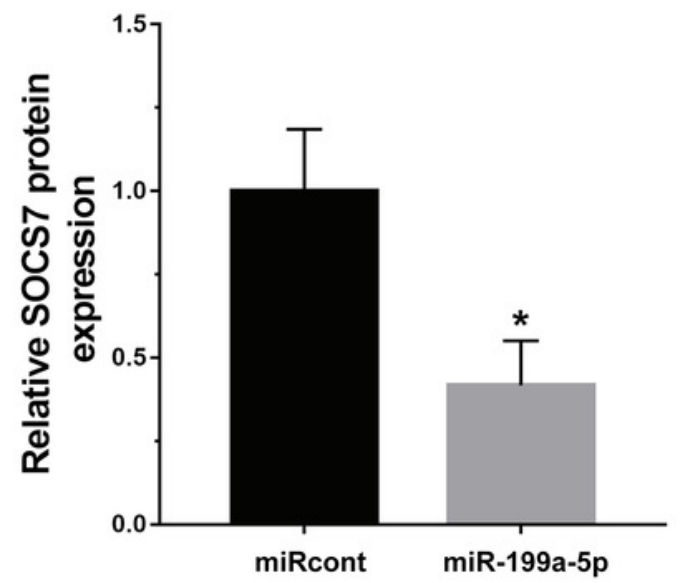




\section{Figure 5}

DZNep repressed liver fibrosis by upregulating SOCS7

( A) The mRNA expression of SOCS7 was detected by qPCR analysis in TGF- $\beta 1$-activated LX2 cells in the presence or absence of DZNep treatment. (B and C) Western blot and quantitative analysis of SOCS7 protein expression in TGF- $\beta 1$-activated LX2 cells in the presence or absence of DZNep treatment. (D-F) The mRNA expression of SOCS7 (D), collagen I (E) and $\alpha \square S M A(F)$ was detected by qPCR analysis in TGF- $\beta 1$-activated LX2 cells in the presence or absence of DZNep treatment after SOCS7 knockdown with siRNA-NC or SOCS7. (G-J) Representative Western blot of SOCS7 (G and H), collagen I ( $G$ and I) and $\alpha \square S M A$ (G and J) in DZNep-treated LX2 cells in the presence of siRNA-NC (siNC) or siSOCS7, and quantification of average across three separate experiments. ${ }^{*} \mathrm{P}<0.05,{ }^{*} \mathrm{P}<0.01$. 


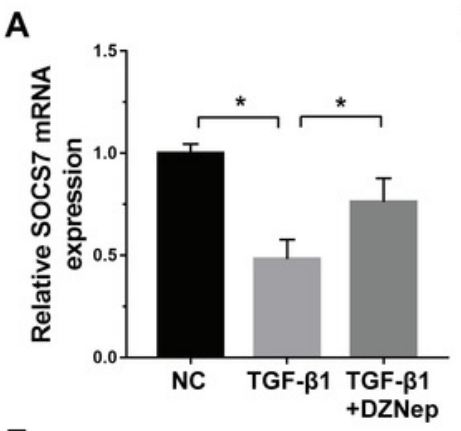

E

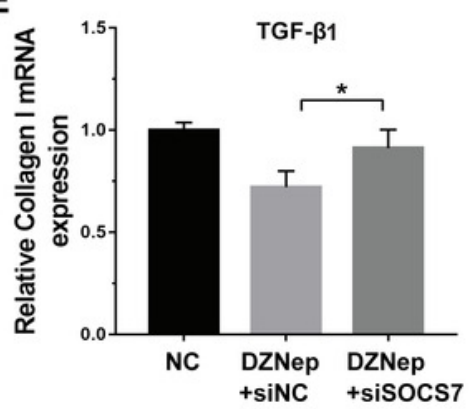

H

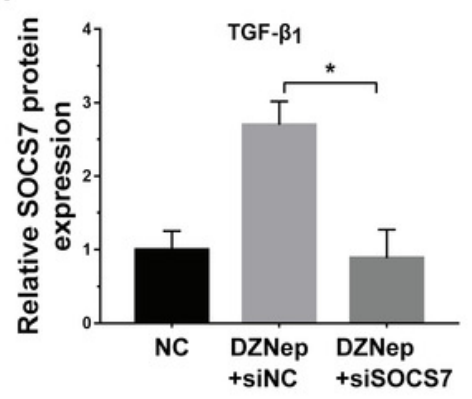

B C

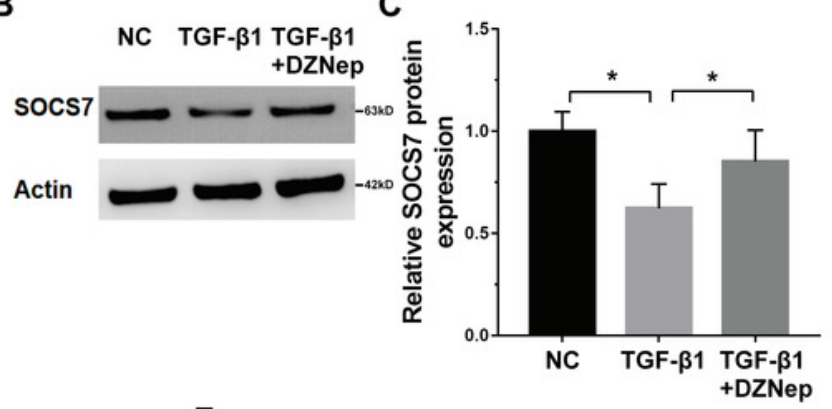

$\mathbf{F}$

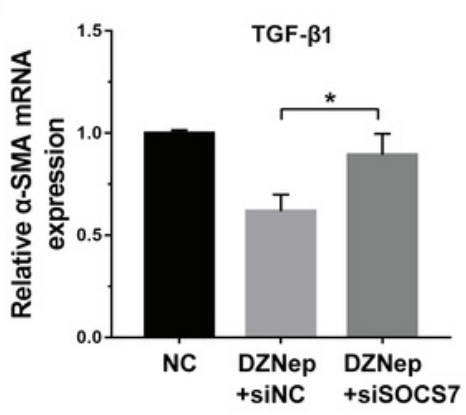

I

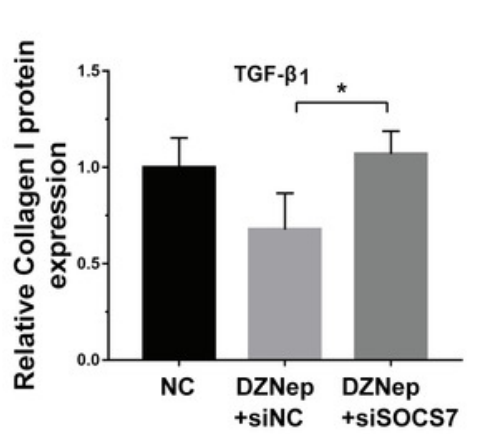

D

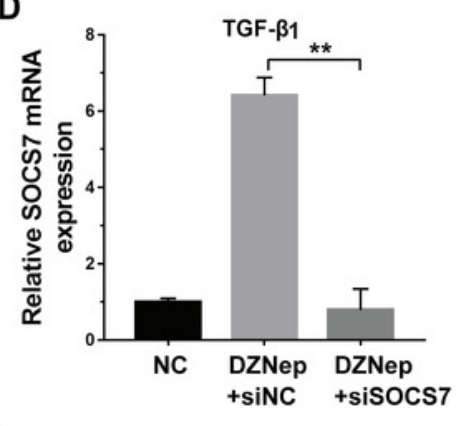

G

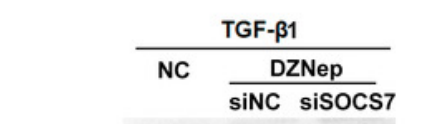

Socs7 - - - swo

Collagen $1+-1300$

a-SMA _- - -

Actin $\longrightarrow=0$

J

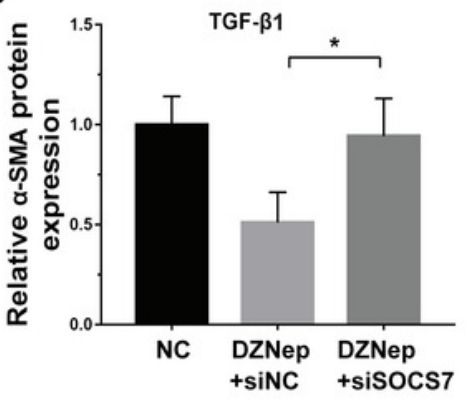

